FORMATION Formation emploi

Revue française de sciences sociales

133 | Janvier-Mars 2016

Heurs et malheurs de l'apprentissage en Suisse

\title{
Quand la transition post-diplôme préfigure les mobilités professionnelles
}

Quatre certifications professionnelles dans les cantons de Vaud et Genève

When the post-graduation transition foreshadows professional mobility : Four professional certifications in the cantons of Vaud and Geneva Wenn der Übergang nach bestandenem Diplom die berufliche Mobilitätvorausahnen lässt : Vier Arten von Berufsbildungsabschlüssen in den Kantonen Waadt und Genf Cuando la transición posdiploma prefigura las movilidades profesionales : cuatro certificaciones profesionales en los cantones de Vaud y Genève

\section{François Rastoldo et Rami Mouad}

\section{(2) OpenEdition}

Journals

Édition électronique

URL : http://journals.openedition.org/formationemploi/4658

DOI : 10.4000/formationemploi.4658

ISSN : 2107-0946

Éditeur

La Documentation française

Édition imprimée

Date de publication : 20 avril 2016

Pagination : $77-100$

ISSN : 0759-6340

Référence électronique

François Rastoldo et Rami Mouad, «Quand la transition post-diplôme préfigure les mobilités professionnelles », Formation emploi [En ligne], 133 | Janvier-Mars 2016, mis en ligne le 21 avril 2018, consulté le 30 octobre 2020. URL : http://journals.openedition.org/formationemploi/4658 ; DOI : https://doi.org/10.4000/formationemploi.4658 


\title{
Quand la transition post-diplôme préfigure les mobilités professionnelles :

\author{
quatre certifications professionnelles dans les \\ cantons de Vaud et Genève
}

\begin{abstract}
FRANÇOIS RASTOLDO Sociologue au Service de la recherche en éducation (SRED) du Département de I'Instruction publique, de la culture et du sport du canton de Genève
\end{abstract}

Rami Mouad Statisticien au Service de la recherche en éducation (SRED) du Département de I'Instruction publique, de la culture et du sport du canton de Genève

Résumé

Quand la transition post-diplôme préfigure les mobilités professionnelles : quatre certifications professionnelles dans les cantons de Vaud et Genève

A partir d'une enquête auprès des diplômés de l'enseignement secondaire II, cet article présente un panorama des transitions vers la vie active et la poursuite d'études des diplômés genevois et vaudois, dix-huit mois après l'obtention d'un diplôme professionnel. Le niveau d'exigence du diplôme et l'organisation de la formation influencent ce processus de transition. Les titulaires d'un certificat fédéral de capacité (CFC) obtenu en alternance connaissent une meilleure insertion sur le marché de l'emploi, alors que les titulaires d'un CFC obtenu en école poursuivent davantage leur formation.

Mots clés : enseignement technique-professionnel, apprentissage, insertion professionnelle, poursuite d'études, cheminement scolaire, certification, Suisse

Abstract

When the post-graduation transition foreshadows professional mobility: Four professional certifications in the cantons of Vaud and Geneva

This article provides an overview of school to work or higher education transitions of young graduates from cantons Geneva and Vaud, eighteen months after their professional diploma. The diploma requirement level as well as the way the training is structured has a clear impact on the nature of the transition. Indeed, apprenticeship graduates with dual (school and enterprise) training have a better integration into the labour market than those with school-only training, while the latter are more likely to pursue their training. 
Keywords: technical \& vocational education, apprenticeship, transition from school to work, continuation of education, school paths, certification, Switzerland

Journal of Economic Literature: I 21, J 62, J 24

Traduction : Auteurs.

Mener l'ensemble des jeunes vers une première certification pour leur permettre, dans les meilleures conditions, une transition vers la vie active ou des études supérieures, représente un enjeu majeur des systèmes de formation.

En Suisse, cet objectif est formalisé par un engagement politique (CDIP, 2011) qui vise à qualifier $95 \%$ des jeunes par un diplôme de niveau secondaire II (niveau de CITE $3^{1}$ ).

La formation professionnelle est au cœur de cette volonté. En effet, elle certifie une grande partie de la jeunesse - $77 \%$ en Suisse et $54 \%$ dans les cantons de Vaud et de Genève (calculé d'après OFS, 2014a) - mais elle prime également dans les stratégies éducatives publiques de formation.

En Suisse, Confédération et cantons mettent en place des politiques publiques de valorisation de la formation professionnelle. Il s'agit d'attirer davantage de jeunes, en arguant qu'une formation professionnelle, surtout duale, permet une bonne articulation entre formation et emploi (DEFR, 2013 ; OFPC, 2013), dimension cruciale en période de tension sur le marché du travail ( $c f$. encadré 2 ).

Cette préoccupation est également présente dans d'autres pays, qu'ils aient une forte tradition de l'apprentissage ou pas. Dans le «Land» de Hesse en Allemagne, une « alliance pour la formation " mise sur un renforcement de la formation professionnelle (IHK Hessen, 2015). En France, le "pacte de responsabilité et de solidarité" prévoit une augmentation des places d'apprentissage (Rebsamen, 2015).

L'observation de la transition entre l'école et la vie active de jeunes dotés d'un diplôme professionnel permet d'évaluer l'articulation entre formation professionnelle, emploi et poursuite d'études supérieures. Cette transition commence, selon l'OCDE (Organisation de coopération et de développement économiques), à l'âge où plus de $75 \%$ de la population est en formation et se termine lorsque la majorité travaille (OCDE, 1996). En Suisse, elle est estimée entre 16 et 21 ans (Padiglia, 2005). Elle comporte deux paliers : la transition I, constituée de l'articulation entre l'école obligatoire et les formations certifiantes de niveau secondaire II et la transition II qui couvre, dès la fin du secondaire II, la transition vers la vie active, en passant par des études supérieures ou non.

1. Classification internationale type de l'enseignement de l'OCDE, qui décrit en termes comparables la structure du système de formation, présentée notamment sur le site : http://www.unige.ch/aref2010/participation/Soumettre/Classification-CITE.pdf 
Cet article se centre sur un moment-clé de cette transition II. Il propose une analyse comparative de la situation des jeunes récemment diplômés de quatre programmes de formation professionnelle, dix-huit mois après l'obtention de leur diplôme, dans deux cantons francophones comptant environ 1,2 millions d'habitants, soit $15 \%$ de la population suisse (OFS, 2014b). Les différents programmes de formation sont hiérarchisés en fonction de leurs exigences (Attestation de formation professionnelle (AFP), Certificat fédéral de capacité (CFC), Maturité professionnelle (MP)) et pour les CFC, différenciés selon leurs modalités d'organisation : duale ou à plein temps en école professionnelle (cf. encadré 2).

Une enquête périodique réalisée dans les cantons de Vaud et de Genève ( $c f$. encadre 1) offre une vision transcantonale de ce moment de transition, dépassant les particularités locales. En effet, si les diplômes sont fédéraux, les modes d'organisation de la formation obligatoire et secondaire II (niveau de CITE 1, 2 et 3) relèvent largement des prérogatives des cantons et sont assez différenciés. Ainsi est-il possible de saisir, au-delà des spécificités locales, à quel point les exigences hiérarchisées des diplômes professionnels affectent la qualité des insertions dans le monde du travail et les opportunités de poursuivre une formation. Il s'agit également de montrer l'effet du choix (parfois contraint) d'une formation duale ou effectuée à plein temps dans une école professionnelle, au niveau du CFC, sur les parcours post-diplômes. Enfin, quelques mesures globales rendent compte des aléas de la période d'insertion qui suit un diplôme professionnel, sous l'angle de la complexité des parcours (directs vs indirects, conduisant à des situations après dix-huit mois estimées stables ou incertaines).

Les éléments de réponse à ces interrogations sont présentés d'abord par un état de la situation des jeunes diplômés dix-huit mois après l'obtention de leur titre. Ensuite, les situations sont analysées sous l'angle de leur linéarité depuis l'obtention du diplôme et de leur stabilité prévisible. Enfin, deux parties sont consacrées à la qualité de l'insertion professionnelle de ceux qui sont en emploi et aux formes de la poursuite d'études de ceux qui poursuivent une formation.

\section{Une problématique de transition}

Plus qu'une période, la transition est un processus, aux contours incertains (Boutinet, 2014), mais limité dans le temps. Ce processus prend en compte l'ensemble des événements de formation et de travail entre la fin de l'école obligatoire et l'emploi (De Broucker, Gensbittel et Mainguet, 2000).

La transition vers la vie active ou vers la poursuite d'études prend forme dans un système de tensions entre aspirations, compétences reconnues et contraintes du contexte. 
C'est à la fois une période durant laquelle l'individu accède à un autre état identitaire et social, de manière volontaire ou subie (Deltand et Kaddouri, 2014) et une composition de contextes sociaux modelant les situations individuelles (Perez-Roux et Balleux, 2014).

\section{Encadré 1 : Méthodologie}

L'enquête source, bi-annuelle, porte sur la situation des diplômés dix-huit mois après l'obtention de leur titre de niveau secondaire II. Elle existe depuis 1989 à Genève et a été étendue pour les diplômés des années 2009 et 2011 au canton de Vaud, dans le cadre d'une collaboration entre le Service de la recherche en éducation du canton de Genève (SRED) et I'Unité de recherche pour le pilotage des systèmes pédagogiques du canton de Vaud (URSP). Elle est réalisée sur une population exhaustive, sauf pour les titulaires du CFC (certificat fédéral de capacité) dans le canton de Vaud, pour lesquels un échantillonnage aléatoire représentant $60 \%$ de la population a été réalisé ( $\mathrm{N}$ total $=11931$ ).

Le questionnaire, stable dans le temps, renseigne : la situation au 1er décembre de l'année qui suit la certification; une évaluation de cette situation; un descriptif des activités effectuées entre la certification et le moment de l'enquête, ainsi qu'une appréciation du futur proche ${ }^{*}$ ). Les informations recueillies sont déclaratives; néanmoins, en cas de poursuite d'études notamment, les réponses ont été confrontées aux registres scolaires pour vérifier leur plausibilité.

Le taux de réponse de 53 \% varie à la marge selon le type de diplôme, plus important chez les titulaires d'un diplôme à exigences élevées, plus bas chez ceux qui ont obtenu une certification à faible qualification. Les filles et les diplômés les plus jeunes tendent également à répondre davantage. Pour tenir compte des non-réponses, une pondération a été réalisée sur quatre critères : le diplôme, le canton, le genre et l'âge. Les données recueillies ont ensuite été croisées avec les bases de données cantonales afin de disposer de variables supplémentaires (description exacte du diplôme et variables sociodémographiques).

Cette enquête permet, pour l'essentiel, une photographie de la situation dix-huit mois après l'obtention du diplôme. L'aspect longitudinal de cette étude réside donc en une comparaison entre un point de départ (le diplôme) et une situation encore largement susceptible d'évoluer (dix-huit mois après). Si les réponses à des questions globales sur les activités exercées durant ces dix-huit mois ont été recueillies, ainsi que des impressions sur la forme du futur proche (vision à une année), une analyse très précise des différentes étapes parcourues durant l'ensemble de cette période n'est pas possible. Ces deux caractéristiques (état à dix-huit mois et vision longitudinale sommaire) constituent les principales limites de cette enquête.

$\left(^{*}\right)$ : Les différentes versions du questionnaire peuvent être consultées sur le site http://www. geneve.ch/recherche-education/eos/quest.asp.

Si les transformations identitaires des jeunes ne sont pas analysées ici, il est important d'avoir à l'esprit qu'elles vont largement modeler cette période, dont les enjeux peuvent être cruciaux pour l'avenir. En effet, c'est le temps de l'apprentissage des rôles d'adulte, de l'autonomie et des responsabilités qui vont avec (Billet et Johnson, 2012) ; une étape de la construction identitaire qui vise tant la singularisation que l'adaptation à l'environnement (Tap, 1988). Durant cette période s'élaborent les projets entre exploration et engagement 
(Marcia, 1966), nécessitant une (re)construction de sens (Masdonati et Zittoun, 2012) qui permet notamment la construction d'une identité professionnelle (Dubar, 2001).

Ce système de tensions, marquant une transition modelée par des choix contextualisés, est représenté ici par le type de diplôme (le choix de la formation, son niveau d'exigence et son mode d'organisation - dual ou à plein-temps en école) ; les caractéristiques sociodémographiques des jeunes ; les exigences et les contraintes du marché du travail ainsi que celles des formations subséquentes. L'articulation entre aspirations, compétences et contraintes n'affecte pas uniquement la destination des jeunes après leur diplôme, mais également les manières d'y parvenir. Pour certains, la transition est un agencement linéaire qui conduit à une insertion souhaitée et satisfaisante. En revanche, pour de nombreux autres, la gestion de ces tensions conduit à des recompositions, parfois itératives, du projet initial, allongeant ainsi la période de transition.

La transition vers la vie active ou les études offre donc des marges de manœuvre durant lesquelles des stratégies individuelles peuvent se développer (Dubet, 1994). Chacun élabore son propre parcours, articulant le souhaitable et le probable (Berthelot, 1993), par des choix susceptibles d'être révisés (Boutinet, 2007) ; ces choix conduisent à de fréquentes bifurcations permettant les adaptations requises par le contexte professionnel multiforme (Guichard, 2013). Ce processus comporte aussi un fort ancrage social qui tend à reproduire les inégalités dans une interaction entre pratiques et structures, conduisant à des carrières socialement différenciées (Bourdieu et Passeron, 1970). Ce processus est donc caractérisé par une "fragmentation marquée » (Keller, Hupka-Brunner et Meyer, 2010, p. 10).

\section{Du dual vers l'emploi et de l'école vers les études}

L'enquête dresse un état des lieux de la situation des diplômés dix-huit mois après l'obtention de leur titre (encadré 1). Elle montre que $59 \%$ des jeunes sont en emploi et $28 \%$ en formation. Le système de formation professionnelle de niveau secondaire II permet effectivement, mais dans des proportions différentes, des orientations plurielles après chacune des certifications qu'il délivre (graphique 1). Le taux de recherche d'emploi, assez conforme aux relevés statistiques nationaux ( $c f$. encadré 2 ), est à la fois modeste en comparaison internationale et notablement plus élevé que le taux de chômage moyen. Les formations duales (CFC dual et AFP) sont plus nettement orientées vers le marché du travail.

Toutefois, après l'AFP, qui certifie des jeunes ayant souvent eu des parcours scolaires difficiles (Hrizi, Mouad, Petrucci et Rastoldo, 2014), les jeunes connaissent un taux de recherche d'emploi élevé. Les formations se déroulant en école (CFC plein-temps et MP pour l'essentiel) conduisent à des orientations plus équilibrées entre poursuite de la formation et accès à emploi. 


\section{Encadré 2 : Le système de formation professionnelle et le marché de l'emploi en Suisse}

Quatre certifications composent le système de formation professionnelle du secondaire II (niveau de CITE 3), décliné en niveaux d'exigences et en métiers spécifiques (*).

Le certificat fédéral de capacité dual (CFC dual) atteste une aptitude professionnelle après trois ou quatre ans d'études, selon les métiers. Cette formation est effectuée en alternance entre une école professionnelle (environ un tiers du temps) qui dispense un enseignement général et spécifique au métier et l'entreprise (deux tiers du temps) qui forme le jeune à l'exercice du métier.

Le Certificat fédéral de capacité effectué à plein-temps en école (CFC plein-temps) s'apparente à celui du système dual. La seule différence réside dans le mode d'apprentissage : les jeunes passent leur temps de formation dans une école professionnelle qui dispense une formation générale, professionnelle et pratique.

L'Attestation fédérale de formation professionnelle (AFP), instaurée en 2004 dans certaines professions, est une formation qui se déroule en deux ans, le plus souvent sur un mode dual. Elle offre aux jeunes qui n'auraient pas les aptitudes scolaires pour effectuer un CFC, une certification de moindre qualification. L'ouverture des AFP à différents métiers répond à une logique d'innovation qui se déroule par étapes, selon les besoins exprimés par les entreprises. L'obtention d'une AFP permet de poursuivre une formation, parfois écourtée, vers un CFC.

La Maturité professionnelle (MP), instaurée en 1993, représente une qualification supplémentaire qui s'additionne au CFC. Elle peut s'obtenir grâce à une année d'étude supplémentaire après le CFC (post-CFC) ou par des cours supplémentaires répartis sur toute la durée du CFC (intra-CFC). La MP permet la poursuite des études au sein des Hautes écoles spécialisées (HES) qui dispensent des formations professionnelles au niveau bachelor et master (niveau de CITE 5). Elle permet également l'accès aux Hautes écoles universitaires (HEU), via une année de formation « passerelle».

L'accès aux formations professionnelles est conditionné par les résultats scolaires, souvent aussi par des tests d'aptitudes lorsque le nombre de places est limité. L'accès aux formations duales nécessite un engagement dans une entreprise formatrice. Les autres formations du secondaire II sont dites " généralistes » et comprennent la filière de la " maturité gymnasiale », permettant l'accès aux HEU, et la filière de " culture générale » qui débouche sur certaines écoles supérieures (ES). Cette dernière filière peut être complétée par une Maturité spécialisée, équivalant à la MP dans les domaines de la santé, du social, de la pédagogie, des arts et de la communication.

Au niveau du marché de l'emploi, Genève et Vaud se caractérisent par un tissu économique tertiarisé (à $81 \%$ dans la région lémanique, OFS, 2014c), qui exige des qualifications élevées et où le diplôme de niveau secondaire II est devenu l'exigence minimale pour accéder à l'emploi dans de bonnes conditions (OFS, 2014d). Comparativement aux pays de l'OCDE (Organisation de coopération et de développement économiques), la Suisse ne connait pas un taux de chômage très élevé : 4.4 \% en 2013 (OFS, 2014e). Cependant, dans les cantons de Genève surtout et de Vaud, dans une moindre mesure, ce taux est supérieur à la moyenne nationale (d'environ 2 points). La transition vers l'emploi reste toujours un moment délicat à négocier pour les jeunes, leur taux de chômage étant environ deux fois plus élevé que la moyenne (8.3 \% en 2013 : OFS, 2014e).

${ }^{*}$ ) : Un schéma de l'organisation de la formation en Suisse peut être consulté sur le site de l'Office fédéral de la statistique : http://www.portal-stat.admin.ch/isced97/docs/G.JB-1520.pdf. 
Environ $7 \%$ des jeunes sont, lors de l'enquête, dans une autre situation. Il s'agit principalement de ceux qui remplissent des obligations citoyennes (service militaire ou civil) ou qui voyagent et effectuent un séjour linguistique. Rares sont ceux qui déclarent une cessation d'activité durable (p. ex. rester à la maison, s'occuper de sa famille, de ses enfants) ou une situation d'attente (problème de santé ou attente d'une reprise de formation). Les jeunes qui relèvent de cette catégorie sont, pour l'essentiel, dans une situation transitoire.

\section{Graphique 1 : Situation des titulaires d'un diplôme professionnel dix-huit mois après l'obtention de leur titre, selon le type de diplôme}

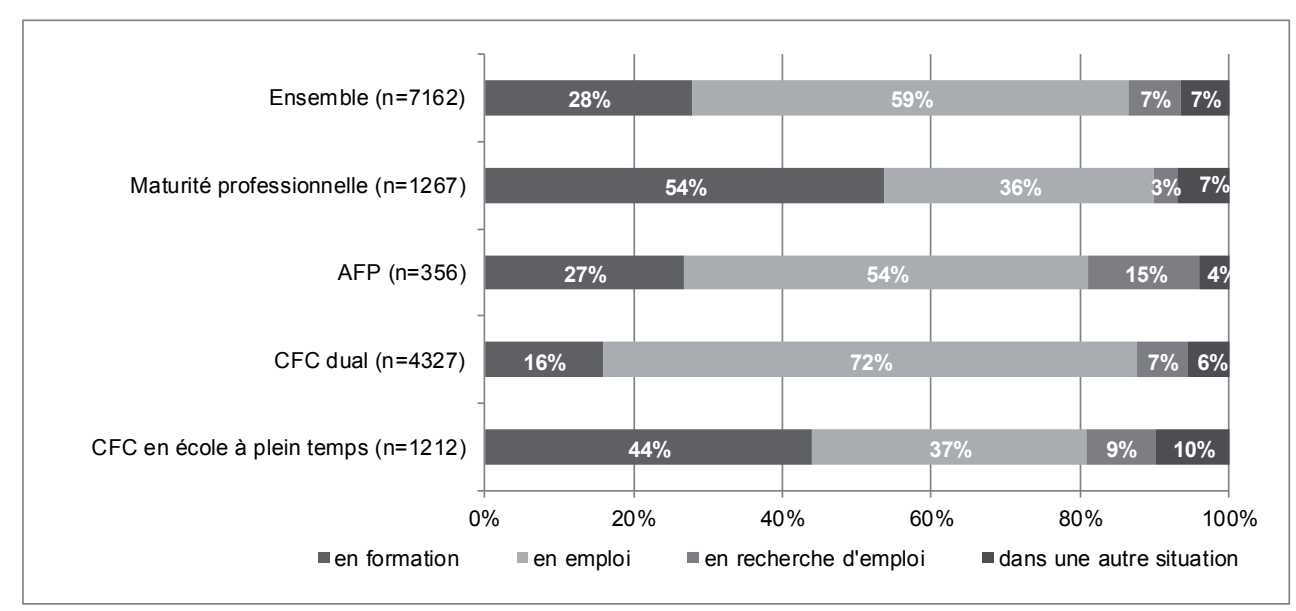

Sigles : AFP : Attestation fédérale de formation professionnelle ; CFC : Certificat fédéral de capacité.

Source : Enquête sur l'orientation des diplômés du secondaire Il.

Une régression logistique multinomiale permet de rendre compte des facteurs qui influencent, toutes choses égales par ailleurs, la situation des jeunes dix-huit mois après leur diplôme entre formation, emploi et recherche d'emploi. ( $c f$. tableau 1). 
Tableau 1 : Chances relatives d'être en emploi, en recherche d'emploi ou en formation, dix-huit mois après l'obtention d'un diplôme professionnel (régression logistique multinomiale)

\begin{tabular}{|c|c|c|c|}
\hline & Variables explicatives & $\begin{array}{c}\text { Chances relatives } \\
\text { (odds ratio) d'être en } \\
\text { formation } \\
\text { (vs en emploi) }\end{array}$ & $\begin{array}{l}\text { Chances relatives } \\
\text { (odds ratio) d'être en } \\
\text { recherche d'emploi } \\
\text { (vs en emploi) }\end{array}$ \\
\hline \multirow[t]{4}{*}{ Diplôme } & Maturité professionnelle & 7,9 & $n s$ \\
\hline & CFC plein-temps & 5,6 & 2,3 \\
\hline & $\mathrm{AFP}$ & 2,3 & 2,7 \\
\hline & CFC dual (réf.) & & \\
\hline \multirow[t]{5}{*}{ Domaine d'activité } & Santé et Social & 1,5 & 0,5 \\
\hline & Artisanat, Services et Restauration & ns & 1,4 \\
\hline & Agriculture et Environnement & 1,5 & $n s$ \\
\hline & Construction et Technique & ns & 0,6 \\
\hline & Commerce (réf.) & & \\
\hline \multirow[t]{3}{*}{ Statut migratoire } & Né en Suisse et allophone & $n s$ & $n s$ \\
\hline & Né ailleurs et allophone & 0,7 & $n s$ \\
\hline & Francophone (réf.) & & \\
\hline \multirow[t]{2}{*}{ Canton } & Genève & 0,8 & 1,4 \\
\hline & Vaud (réf.) & & \\
\hline \multirow[t]{2}{*}{ Genre } & Masculin & 1,5 & 1,4 \\
\hline & Féminin (réf.) & & \\
\hline \multirow[t]{2}{*}{ Age } & 20 ans et moins & 1,7 & 0,8 \\
\hline & Plus de 20 ans (rèf.) & & \\
\hline
\end{tabular}

Clé de lecture pour les tableaux 1 à $3:$ Un odds ratio > 1 signifie un accroissement de l'occurrence de l'évènement observé par rapport à la situation de référence. Un odds ratio $<1$ signifie la diminution de cette occurrence.

Exemple de lecture : «Toutes choses égales par ailleurs », les titulaires d'un CFC plein-temps ont 5,6 fois plus de chances d'être en formation qu'en emploi, par rapport à ceux qui ont un CFC dual (référence).

Source : Enquête sur l'orientation des diplômés du secondaire II.

Le domaine d'activité module la situation². Les diplômés du domaine "santé-social " sont à la fois plus nombreux à poursuivre une formation supérieure, indispensable pour nombre de professions sociales et médicales (éducateurs, infirmiers notamment), et relativement moins touchés par le chômage en raison d'une offre d'emploi importante dans ce secteur. Pour les mêmes raisons, le risque de chômage est moindre dans le domaine de la construction et des professions techniques, alors qu'il est majoré dans les domaines de l'artisanat, des services et de la restauration qui connaissent un taux de chômage quasiment deux fois plus important que la moyenne Suisse (OFS, 2014e).

La proportion de jeunes passant par la formation professionnelle est plus importante dans le canton de Vaud ( $c f$. introduction) et ils poursuivent plus souvent une formation après

2. Cinq domaines d'activité ont été retenus : santé-social, commerce, technique et construction, artisanatservices-restauration et agriculture-environnement. Cette classification résulte de la mise en commun des classifications, en partie différentes, utilisées dans les administrations genevoise et vaudoise. 
un diplôme professionnel. En fait, dans le canton de Genève, la poursuite d'études vs l'entrée dans la vie active est davantage liée au type de diplôme (la Maturité gymnasiale surtout). Dans le canton de Vaud, où le taux de maturité gymnasiale est plus faible, les voies vers des études supérieures sont plus diversifiées, notamment via les diplômes professionnels (Bachmann Hunziker et al., 2014). Vaud et Genève se différencient également par leur marché du travail, moins tendu dans le canton de Vaud où les risques de chômage sont plus faibles ( $c f$. encadré 2).

Les plus jeunes (20 ans ou moins) sont plus fréquemment en formation et moins souvent en recherche d'emploi que les diplômés plus âgés (plus de 20 ans). Les plus âgés ont connu, avant leur diplôme, une formation prolongée, que ce soit par des redoublements durant leur scolarité antérieure, par des réorientations durant les études secondaires (changement de filière, d'apprentissage), ou parce qu'ils ont interrompu momentanément leur formation avant l'obtention du diplôme. Ces trois éléments relèvent en grande partie de difficultés scolaires antérieures qui les ont empêchés de terminer leur formation dans les temps (Hrizi et al., 2014). Cette différence de niveau, que révèle l'âge des diplômés, limite à la fois leurs possibilités de poursuite d'études et leurs chances d'accéder à l'emploi.

Les jeunes migrants (identifiés ici par un statut d'allophone et une arrivée dans les cantons de Genève ou de Vaud après leur naissance) tendent à poursuivre moins souvent des études que les autochtones francophones, ce qui n'est pas le cas des jeunes migrants de la seconde génération (allophones nés dans les cantons de Genève ou de Vaud). L'origine sociale, qui n'est pas présentée dans le tableau 1, mais mesurée pour le canton de Genève ${ }^{3}$, montre que les jeunes issus des milieux moins favorisés privilégient l'accès à l'emploi, alors que ceux issus des milieux les plus favorisés poursuivent plus fréquemment des études après le diplôme professionnel ${ }^{4}$.

Le statut migratoire, de même que l'origine sociale mesurée pour Genève n'apparaissent pas significatifs dans la mesure du risque de chômage, toutes chose égales par ailleurs. En revanche, une variable de l'enquête source mesurant le temps nécessaire pour l'obtention du premier emploi vient nuancer cette observation, assez sommaire. En effet, les jeunes immigrés ont rencontré plus de difficultés que les autochtones francophones pour décrocher ce premier emploi $(80 \%$ vs $70 \%$ qui obtiennent leur premier emploi en trois mois ou moins). Ce résultat corrobore ceux d'autres études qui ont mis en évidence des discriminations à l'embauche (Fibbi, Kaya et Piguet, 2003).

3. Lorigine sociale définie par une catégorie socioprofessionnelle (CSP) n'est disponible, dans les registres scolaires, que dans le canton de Genève. Son influence a été mesurée pour les Genevois ; il n’a en revanche pas été possible d'en tenir compte dans les analyses regroupant les diplômés genevois et vaudois.

4. Les chances relatives d'être en formation pour la population genevoise sont respectivement de 0.5 pour les jeunes issus des milieux défavorisés et de 1.4 pour les jeunes issus des milieux les plus favorisés (référence classe moyenne). 
Les femmes s'orientent davantage vers le marché du travail après une formation professionnelle. Outre le fait connu que les formations professionnelles sont aussi genrées que les métiers auxquels elles préparent, les filières de formation professionnelle comptent davantage de jeunes hommes qui choisissent cette orientation afin de poursuivre des études supérieures. Les jeunes femmes, à niveau équivalent et qui souhaitent poursuivre une formation supérieure, choisissent plus souvent les filières scolaires généralistes, notamment la filière gymnasiale. Le chômage semble également moins important pour les jeunes femmes titulaires d'un diplôme professionnel. Ce résultat diffère des tendances globales du marché du travail qui révèlent des taux de recherche d'emploi relativement équiprobables, selon le genre, chez les jeunes de moins de 25 ans (OFS, 2014e).

La formation professionnelle participe donc, au même titre que les autres filières de formation, à la reproduction des parcours de formation selon la situation sociale (Lamamra, Fassa Recrosio et Chaponnière, 2014).

\section{Des formes de transition post-diplôme 3) rarement linéaires}

Dix-huit mois après le diplôme, le processus de transition est encore largement en cours (graphiques 2 et $\mathbf{3}$ ). A peine plus d'une moitié $(55 \%)$ des jeunes qui exercent un emploi ou poursuivent une formation ont accédé directement à l'activité qu'ils exercent au moment de l'enquête (transition directe).

En se projetant vers l'avenir, moins de la moitié des diplômés pensent que leur situation au moment de l'enquête va se maintenir toute l'année. Au total, seul un tiers des jeunes connaît un parcours direct entre son diplôme et sa situation après dix-huit mois et pense que cette dernière sera stable l'année suivante.

La transition directe ou indirecte à la situation dix-huit mois après le diplôme et l'estimation de la stabilité de l'avenir proche confèrent une dimension dynamique à la transition que les jeunes conduisent par des négociations entre le prescrit et leurs aspirations (Doray et al., 2009a) ; la linéarité de cette transition est souvent plus théorique qu'effective, en raison de réajustements continus (Padiglia, 2007). Si certains parcours de formation linéaires persistent, les parcours atypiques ou indirects sont nombreux, que ce soit pour parvenir à l'obtention d'un premier diplôme (Pollien et Bonoli, 2012) ou pour transiter vers le marché du travail (Bachmann Hunziker et al., 2014). 
Graphique 2 : Transition directe ou non à la situation de formation ou d'emploi dix-huit mois après le diplôme et selon le diplôme

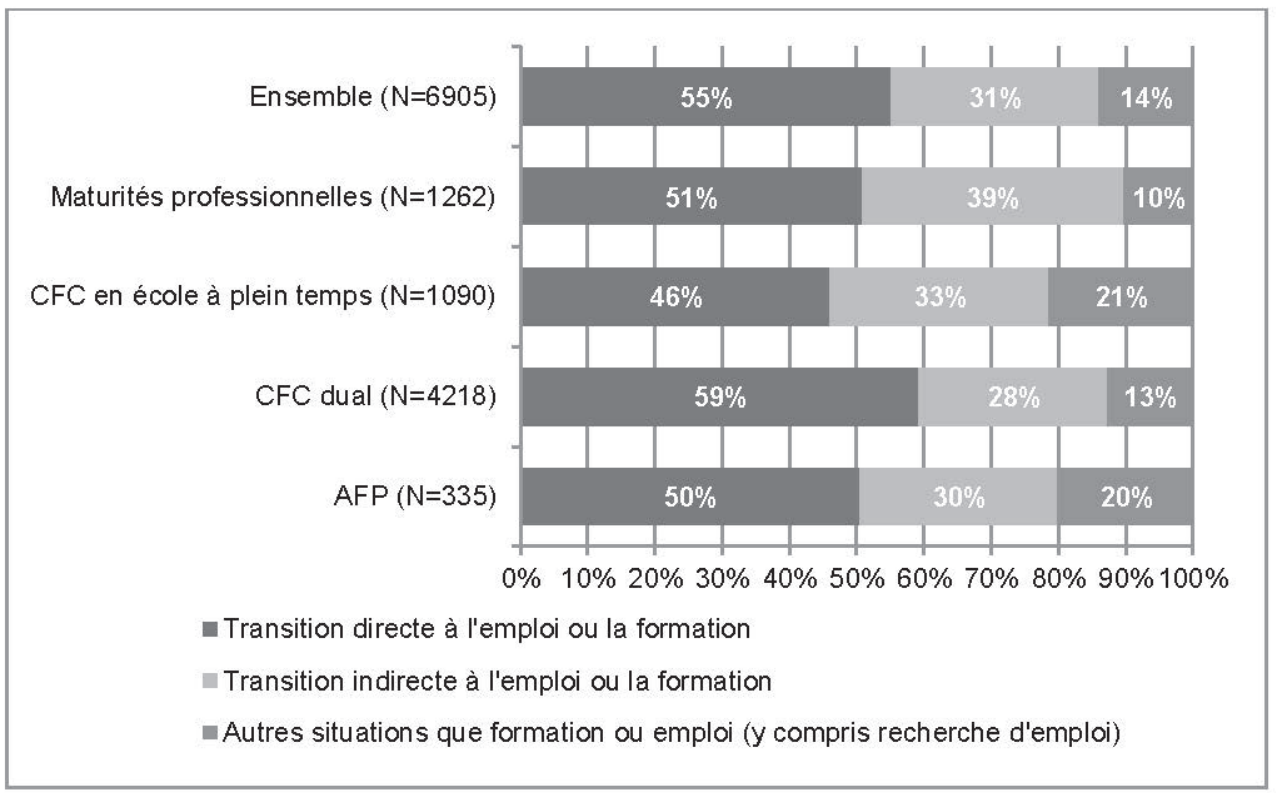

Graphique 3 : Anticipation, au moment de l'enquête, de la situation pour l'année à venir, selon le diplôme

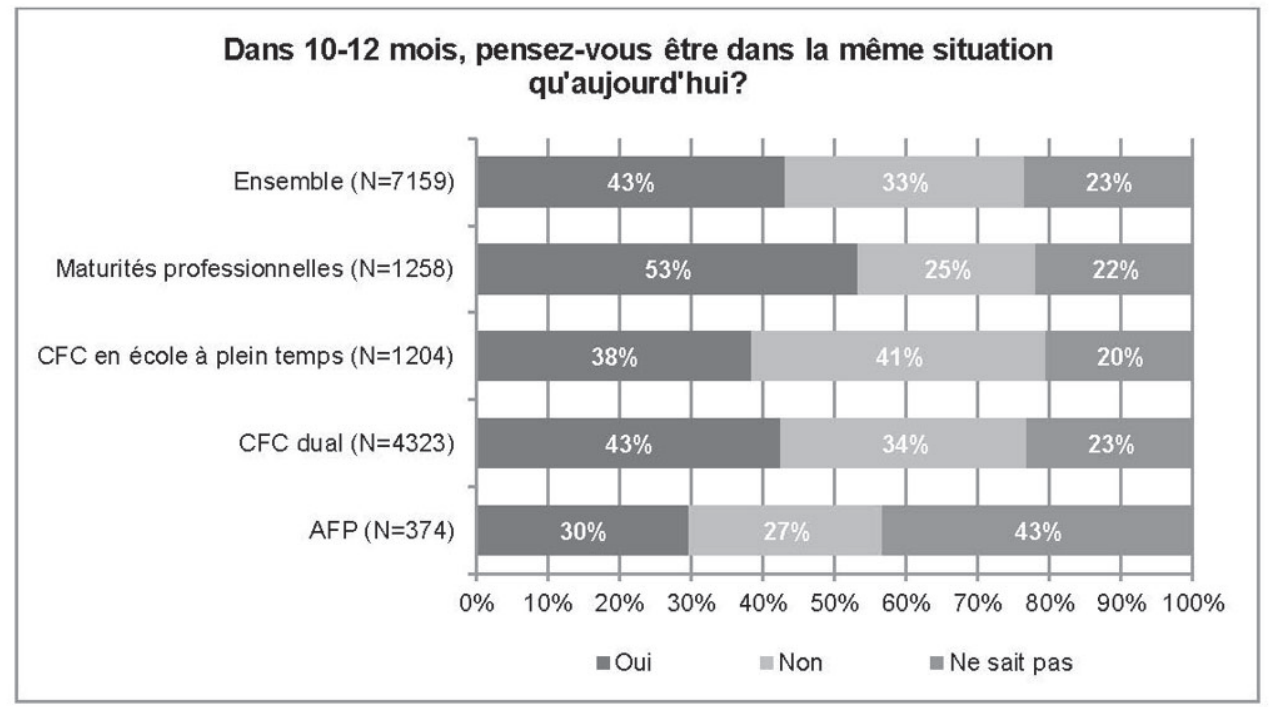

Sigles : AFP : Attestation fédérale de formation professionnelle ; CFC : certificat fédéral de capacité.

Exemple de lecture : 59 \% des titulaires d'un CFC dual ont accédé directement, après leur diplôme, à l'emploi ou à la formation qu'ils occupent dix-huit mois après.

Source : Enquête sur l'orientation des diplômés du secondaire II 
Une régression logistique portant sur les chances relatives de connaitre une transition indirecte à la situation dix-huit mois après le diplôme (tableau 2) montre que la poursuite d'une formation est davantage indirecte que l'accès à l'emploi. Les jeunes ayant obtenu un CFC en école connaissent également des transitions plus complexes. Non seulement ces derniers se partagent assez équitablement entre études et emploi (graphique 1), mais le font davantage par des essais de l'un et de l'autre. Les domaines d'activité ont un effet corrélé avec le risque de chômage. Les domaines où ce risque est minoré («Santé et Social » et "Construction et Technique ») connaissent des transitions directes plus fréquentes.

Tableau 2 : Chances relatives de connaître une transition indirecte entre le diplôme et la situation d'emploi ou de formation dix-huit mois après (régression logistique).

\begin{tabular}{|c|c|c|}
\hline & ariables explicatives & $\begin{array}{l}\text { Chances relatives (odds } \\
\text { ratio) de connaître une } \\
\text { transition indirecte à } \\
\text { l'emploi ou à la } \\
\text { formation } \\
\text { ( } v s \text { directe) }\end{array}$ \\
\hline \multirow[t]{2}{*}{ Activités à 18 mois } & en formation & 2,8 \\
\hline & en emploi (réf.) & \\
\hline \multirow[t]{2}{*}{ Diplôme } & $\begin{array}{l}\text { Maturité professionnelle } \\
\text { CFC plein-temps } \\
\text { AFP }\end{array}$ & $\begin{array}{l}n s \\
1,3 \\
n s\end{array}$ \\
\hline & $C F C$ dual (réf.) & \\
\hline Domaine d'activité & $\begin{array}{l}\text { Santé et Social } \\
\text { Artisanat, Services et Restauration } \\
\text { Agriculture et Environnement } \\
\text { Construction et Technique } \\
\text { Commerce (réf.) }\end{array}$ & $\begin{array}{l}0,8 \\
n s \\
0,7 \\
0,7\end{array}$ \\
\hline \multirow[t]{2}{*}{ Statut migratoire } & $\begin{array}{l}\text { Né en Suisse et allophone } \\
\text { Né ailleurs et allophone }\end{array}$ & $\begin{array}{l}0,8 \\
n s\end{array}$ \\
\hline & Francophone & \\
\hline Canton & $\begin{array}{l}\text { Genève } \\
\text { Vaud (réf.) }\end{array}$ & $n s$ \\
\hline Genre & $\begin{array}{l}\text { Masculin } \\
\text { Féminin(réf.) }\end{array}$ & 1,2 \\
\hline Age & $\begin{array}{l}20 \text { ans et moins } \\
\text { Plus de } 20 \text { ans (réf.) }\end{array}$ & 1,2 \\
\hline
\end{tabular}

Sigles : AFP : Attestation fédérale de formation professionnelle; CFC : Certificat fédéral de capacité.

Exemple de lecture : " Toutes choses égales par ailleurs », les titulaires d'un CFC plein-temps ont 1,3 fois plus de chances de connaitre une transition indirecte à l'emploi ou à la formation qu'ils occupent, par rapport à ceux qui ont un (FC dual (référence).

Source : Enquête sur l'orientation des diplômés du secondaire Il. 
Les plus âgés, qui ont obtenu un diplôme après un parcours de formation déjà émaillé de redoublements, de réorientations ou d'interruptions temporaires de leur formation, ont davantage tendance à rechercher une insertion durable. Quant aux plus jeunes, ils peuvent encore, après le diplôme, tester diverses voies d'insertion (segments de formation, périodes d'emploi). En effet, le diplôme n'intervient pas pour tous au même moment de la période de transition vers la vie active.

La fin de la formation professionnelle constitue aussi le moment où un grand nombre de jeunes vont accomplir leur service militaire ou civil. Cette obligation ne concerne que les jeunes hommes (elle est facultative pour les jeunes femmes) de nationalité suisse. Cela explique que les femmes et les jeunes issus de l'immigration (souvent de nationalité étrangère en raison d'un code de la nationalité assez restrictif en Suisse) accèdent plus souvent directement à la situation qu'ils connaissent dix-huit mois après le l'obtention du diplôme.

La vision des diplômés pour l'année à venir conforte ces résultats (tableau 3). Les jeunes en formation ont une vision plus stable de leur futur proche, alors que ceux qui recherchent un emploi ou qui exercent d'autres activités sont davantage dans l'incertitude et l'anticipation d'un changement. La Maturité professionnelle semble également permettre une vision de l'avenir moins changeante, contrairement aux titulaires d'une AFP dont le niveau d'incertitude face à l'avenir est fort. Les domaines "Santé et Social " et "Construction et Technique " sont également ceux qui offrent les plus grandes perspectives de stabilité à court terme.

Si les jeunes issus de l'immigration (allophone nés en Suisse) ont des parcours plus linéaires après leur diplôme, leur degré d'incertitude face à l'avenir est un peu plus élevé.

Enfin, les diplômés les plus jeunes sont davantage dans une perspective de changement, confirmant leur positionnement différent dans le processus de transition vers la vie active. 
Tableau 3 : Chances relatives d'estimer une situation stable, changeante ou incertaine à un an terme (régression logistique multinomiale)

\begin{tabular}{|c|c|c|c|}
\hline & Variables explicatives & $\begin{array}{l}\text { Chances relatives (odds } \\
\text { ratio) d'envisager } \\
\text { l'avenir avec incertitude } \\
\text { (vs situation stable) }\end{array}$ & $\begin{array}{l}\text { Chances relatives (odds } \\
\text { ratio) d'anticiper un } \\
\text { changement de situation } \\
\text { dans l'année à venir } \\
\text { (vs situation stable) }\end{array}$ \\
\hline \multirow[t]{2}{*}{ Activité à 18 mois } & $\begin{array}{l}\text { en formation } \\
\text { en recherche d'emploi } \\
\text { dans une autre situation }\end{array}$ & $\begin{array}{l}0,6 \\
12,5 \\
2,7\end{array}$ & $\begin{array}{l}n s \\
40,2 \\
15,9\end{array}$ \\
\hline & en emploi (réf.) & & \\
\hline \multirow[t]{2}{*}{ Diplôme } & $\begin{array}{l}\text { Maturité professionnelle } \\
\text { CFC plein-temps } \\
\text { AFP }\end{array}$ & $\begin{array}{l}n s \\
n s \\
2,3\end{array}$ & $\begin{array}{l}0,6 \\
n s \\
n s\end{array}$ \\
\hline & CFC dual (réf.) & & \\
\hline \multirow[t]{2}{*}{ Domaine d'activité } & $\begin{array}{l}\text { Santé et Social } \\
\text { Artisanat, Services et Restauration } \\
\text { Agriculture et Environnement } \\
\text { Construction et Technique }\end{array}$ & $\begin{array}{l}0,8 \\
n s \\
n s \\
0,7\end{array}$ & $\begin{array}{l}0,8 \\
n s \\
0,5 \\
0,6\end{array}$ \\
\hline & Commerce (réf.) & & \\
\hline \multirow[t]{2}{*}{ Statut migratoire } & $\begin{array}{l}\text { Né en Suisse et allophone } \\
\text { Né ailleurs et allophone }\end{array}$ & $\begin{array}{l}1,3 \\
n s\end{array}$ & $\begin{array}{l}n s \\
n s\end{array}$ \\
\hline & Francophone (rèé.) & & \\
\hline \multirow[t]{2}{*}{ Canton } & Genève & ns & 0,9 \\
\hline & Vaud (rèé.) & & \\
\hline \multirow[t]{2}{*}{ Genre } & Masculin & ns & $n s$ \\
\hline & Féminin (réf.) & & \\
\hline \multirow[t]{2}{*}{ Age } & 20 ans et moins & $n s$ & 1,5 \\
\hline & Plus de 20 ans (rèf.) & & \\
\hline
\end{tabular}

Sigles : AFP : Attestation fédérale de formation professionnelle ; CFC : certificat fédéral de capacité.

Exemple de lecture : " Toutes choses égales par ailleurs », les titulaires d'une AFP ont 2,3 fois plus de chances d'envisager l'avenir avec incertitude, par rapport à ceux qui ont un CFC dual (référence).

Source : Enquête sur l'orientation des diplômés du secondaire II.

Les dix-huit mois qui suivent le diplôme sont riches de réorientations, essais et période de latence (recherche d'emploi notamment) et composent des transitions souvent complexes (Davaud, Mouad et Rastoldo, 2010 ; Cattaneo, Donati et Galeandro Bocchino, 2009 ; Meyer, Hupka-Brunner et Keller, 2012). En cela, la Suisse ne constitue pas une exception. Le Canada compte, au sein de son système de formation, seulement $45 \%$ de parcours continus (Doray et al., 2009b). L'Angleterre connait également une diversité grandissante des parcours de formation de sa jeunesse (Bloomer et Hodkinsond, 2000). En France, un jeune sur quatre échappe au modèle de carrière proposé par l'institution scolaire (Boudesseul et al., 2008) et moins de six jeunes sur dix connaissent une transition rapide et durable vers l'emploi (Céreq, 2012). 


\section{L'expérience de l'entreprise facilite la qualité de l'insertion professionnelle}

Les niveaux de chômage échelonnés selon l'exigence du diplôme (graphique 1) fournissent une indication sur la qualité de l'insertion professionnelle. Cependant, cette dernière doit être encore évaluée à l'aune de l'adéquation entre emploi et formation, des conditions de travail et de la satisfaction des jeunes (Beduwé et Fourcade, 2014).

Le CFC dual, davantage tourné vers l'emploi (graphique 1), dans le cas d'une transition souvent directe (tableau 2), avec des risques de chômage faibles, permet également une bonne qualité d'intégration professionnelle (tableau 4). Les conditions de travail précaires sont plus rares, et la satisfaction vis-à-vis du travail exercé est plutôt meilleure. Cette transition à l'emploi relativement aisée tient au fait que ces jeunes sont déjà insérés dans une entreprise depuis trois ou quatre ans et qu'une partie des difficultés d'intégration que rencontrent les jeunes sortant de l'école sont connues et ont été déjà surmontées durant l'apprentissage. Cette transition est d'autant plus facile que $30 \%$ environ des jeunes titulaires d'un CFC dual poursuivent leurs activités, en qualité d'employé ou d'ouvrier qualifié, dans l'entreprise qui les a formés. Cela signifie que l'apprentissage dual représente également une modalité de gestion des ressources humaines pour certaines entreprises qui forment et sélectionnent ainsi les personnels dont elles auront besoin.

Après un CFC plein-temps, l'intégration dans la vie active est délicate, avec un taux de recherche d'emploi plus élevé et des passages par des statuts précaires plus fréquents. Ces caractéristiques reflètent les ajustements nécessaires lors du passage de l'école à l'emploi, même si l'école est fortement professionnalisée. Ce constat doit cependant être nuancé, puisque près de la moitié ( $44 \%$ ) des jeunes obtenant un CFC plein-temps poursuivent leur formation, ce qui les positionnera différemment sur le marché du travail.

Bien que l'AFP se déroule, dans $95 \%$ des cas, en alternance, l'accès à l'emploi est plus difficile que pour les titulaires d'un CFC dual. Le taux de recherche d'emploi est assez élevé, et les conditions d'emploi précaires sont plus nombreuses. Ces éléments témoignent de la relative difficulté à entrer dans la vie active avec un diplôme peu qualifiant. 
Tableau 4 : Indicateurs de la qualité de l'emploi exercé

\begin{tabular}{|c|c|c|c|c|c|}
\hline & AFP & CFC dual & $\begin{array}{l}\text { CFC plein- } \\
\text { temps }\end{array}$ & MP & Ensemble \\
\hline \multicolumn{6}{|l|}{ 1) Qualité de l'emploi } \\
\hline Contrats à durée déterminée & $16 \%$ & $10 \%$ & $24 \%$ & $22 \%$ & $13 \%$ \\
\hline Temps de travail inférieur à 25 heures hebdomadaires & $18 \%$ & $4 \%$ & $7 \%$ & $11 \%$ & $5 \%$ \\
\hline Emploi de stagiaires, auxiliaires et employés non qualifiés & $24 \%$ & $7 \%$ & $15 \%$ & $22 \%$ & $10 \%$ \\
\hline \multicolumn{6}{|l|}{ 2) Qualité du travail } \\
\hline Qualification adéquate à la formation & $72 \%$ & $70 \%$ & $62 \%$ & $54 \%$ & $68 \%$ \\
\hline Adéquation à la formation (échelle de 1à 9) & 7.1 & 7.7 & 6.8 & 6.2 & 7.4 \\
\hline Satisfaction à l'égard de la rémunération (échelle de 1 à 9) & 6.1 & 5.9 & 6.1 & 5.6 & 5.9 \\
\hline Satisfaction à l'égard du travail à effectuer (échelle de 1 à 9) & 7.3 & 7.2 & 6.8 & 6.7 & 7.1 \\
\hline $\begin{array}{l}\text { Satisfaction à l'égard des perspectives de carrière (échelles } \\
\text { de 1 à 9) }\end{array}$ & 6.6 & 6.2 & 6 & 6.2 & 6.2 \\
\hline $\begin{array}{l}\text { Satisfaction à l'égard des horaires de travail (échelle de } 1 \\
\text { à 9) }\end{array}$ & 7 & 6.8 & 6.9 & 6.5 & 6.8 \\
\hline \multicolumn{6}{|l|}{ 3) Appréhension de l'avenir } \\
\hline $\begin{array}{l}\text { Vision de l'avenir (de } 1 \text { « très défavorable » à } 9 \text { « très } \\
\text { favorable ») }\end{array}$ & 6.5 & 6.5 & 6.6 & 7.1 & 6.6 \\
\hline N max (pondéré) & 194 & 3108 & 446 & 458 & 4206 \\
\hline
\end{tabular}

Clé de lecture : Bien que les dimensions décrites soient largement factuelles, il s'agit de mesures déclaratives qui reflètent la manière dont les jeunes les relatent. Pour l'ensemble des indices, le N peut varier de quelques unités en fonction des non-réponses. Seuil de significativité de $5 \%$.

Sigles : AFP : Attestation de formation professionnelle ; CFC : Certificat fédéral de capacité ; MP : Maturité professionnelle.

Exemple de lecture : Un emploi d'une durée hebdomadaire inférieure à 25 heures concerne $5 \%$ de l'ensemble des diplômés, mais $18 \%$ des titulaires d'une AFP.

Source : Enquête sur l'orientation des diplômés du secondaire II.

Pour les titulaires d'une AFP qui travaillent, on observe cependant une bonne adéquation entre qualifications et emploi, ainsi que la satisfaction à l'égard du travail et des perspectives de carrière. Cette posture doit être interprétée en regard de leur parcours scolaire global, qui a été assez difficile et ne leur a pas permis de suivre une formation de niveau CFC. Aussi, le fait d'être certifié et d'avoir un emploi est en soi un motif de satisfaction, même si, dans les faits, les perspectives sont plus limitées, notamment en termes de revenus (environ $12 \%$ moins élevés que ceux des titulaires d'un CFC ; Kammermann, Balzer et Hättich, 2013) et de vision d'avenir, qu'ils jugent plus incertain.

La MP, initialement conçue comme un complément de formation devant permettre l'accès aux HES, est aussi largement utilisée comme un atout supplémentaire pour l'emploi. Ce que le système de formation reconnaît pour accéder aux études supérieures, les entreprises le reconnaissent également comme gage d'employabilité. Ainsi, en plus d'un risque de chômage faible, à dix-huit mois, l'avenir est envisagé favorablement, mais au prix d'une situation d'emploi un peu plus souvent précaire et des attentes visiblement moins satisfaites concernant le travail. Ce contraste illustre une transition vers la vie 
active aisée et qui semble prometteuse ; en revanche, elle nécessite du temps et de nombreux ajustements.

D’une manière générale, les jeunes les moins satisfaits de leur emploi sont aussi ceux qui pressentent un changement de leur situation (tableau 5).

Tableau 5 : Différences de vision d'avenir, selon le degré de satisfaction au travail

\begin{tabular}{|l|c|c|}
\cline { 2 - 3 } \multicolumn{1}{c|}{} & \multicolumn{1}{c|}{$\begin{array}{c}\text { Même situation } \\
\text { dans un an }\end{array}$} & $\begin{array}{c}\text { Changement de situation dans } \\
\text { I'année }\end{array}$ \\
\hline Adéquation à la formation (échelle de 1à 9) & 7.8 & 6.9 \\
\hline $\begin{array}{l}\text { Satisfaction à l'égard de la rémunération (échelle de } \\
\text { 1à 9) }\end{array}$ & 6.4 & 5.3 \\
\hline $\begin{array}{l}\text { Satisfaction à l'égard du travail à effectuer (échelle } \\
\text { de 1 à 9) }\end{array}$ & 7.7 & 6.1 \\
\hline $\begin{array}{l}\text { Satisfaction à l'égard des perspectives de carrière } \\
\text { (échelle de 1à 9) }\end{array}$ & 7.1 & 4.8 \\
\hline $\begin{array}{l}\text { Satisfaction à l'égard des horaires de travail (échelle } \\
\text { de 1à 9) }\end{array}$ & 7.4 & 6.1 \\
\hline
\end{tabular}

Analyse de la variance $p<0.05$.

Source : Enquête sur l'orientation des diplômés du secondaire II.

La qualité de l'emploi est un élément qui favorise la stabilité. A l'inverse, ceux qui jugent leur situation insatisfaisante anticipent plus souvent un changement les menant vers d'autres formes de transition afin de mieux ajuster leur situation à leurs aspirations, que ce soit par un changement d'emploi ou un retour en formation.

\section{Les poursuites d'études, entre ouverture vers le supérieur et requalification}

Formellement, il est possible d'effectuer un parcours complet dans le système de formation professionnelle du secondaire II, une AFP suivie d'un CFC, ensuite une MP pour continuer une formation professionnelle supérieure (HES). Cette enquête ne permet pas de repérer de tels cursus; cependant, une analyse des registres scolaires genevois sur les dix dernières années ${ }^{5}$ révèle que le parcours de l'AFP à une HES n'a jamais été réalisé. Ce résultat n'est pas surprenant dans la mesure où les jeunes qui entreprennent une AFP le font en raison de difficultés scolaires majeures.

LAFP prend donc place entre qualification minimale en vue d'un accès à l'emploi pour ceux dont les difficultés scolaires ne permettent pas une certification plus exigeante, et

5. Analyse des informations de la base de données scolaires du canton de Genève relevant les situations scolaires au 31.12 de chaque année. 
préparation au CFC pour ceux dont les difficultés sont probablement moins importantes. La corrélation positive entre le fait de ne pas poursuivre de formation après l'AFP et celui d'être passé par l'enseignement spécialisé ${ }^{6}$ (V de Cramer 0.25, p<0.01) illustre cette dualité. Au final, $27 \%$ poursuivent un cursus de qualification visant l'obtention d'un CFC, et ce taux est plutôt en augmentation (18\% en 2007, $27 \%$ en 2009 et $41 \%$ en 2011 sur la base des données genevoises), positionnant l'AFP de plus en plus comme une propédeutique au CFC.

Après un CFC, la poursuite de la formation est fortement différenciée selon la modalité d'obtention du CFC (graphique 4).

\section{Graphique 4 : Parcours des jeunes, 18 mois après l'obtention de leur CFC en école à plein-temps et dual}

\section{Après un CFC plein temps}

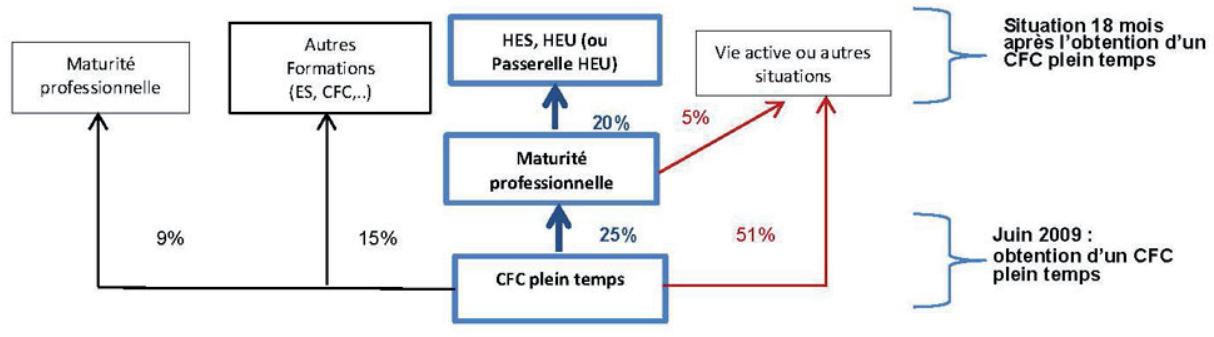

\section{Après un CFC dual}

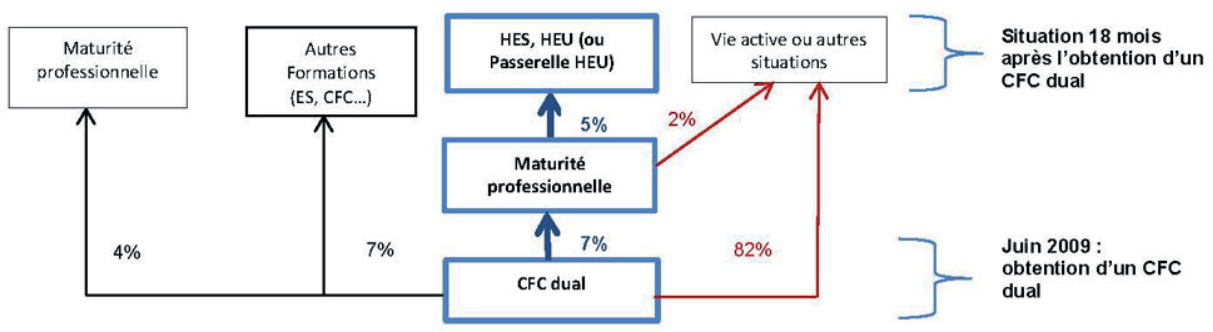

Sigles : CFC : certificat fédéral de capacité; HES : hautes écoles spécialisées ; HEU : hautes écoles universitaires ; ES : écoles supérieures.

Exemple de lecture : $7 \%$ des titulaires d'un CFC dual obtiennent une maturité professionnelle directement l'année suivante. $5 \%$ poursuivent ensuite, dix-mois après le diplôme, des études vers des Hautes écoles et $2 \%$ se dirigent, après leur Maturité professionnelle, vers le marché de l'emploi. Source : Enquête sur l'orientation des diplômés du secondaire II.

6. L'enseignement spécialisé concerne les élèves présentant des handicaps et/ou de grandes difficultés d'apprentissage. Par ailleurs, une étude genevoise décrivant les parcours antérieurs à l'AFP met en évidence les difficultés scolaires de ces jeunes, comme les redoublements, les réorientations ou les passages par des structures passerelles entre le secondaire I et II (Hrizi et al., 2014). 
Parmi les titulaires d'un CFC plein-temps, $20 \%$ ont effectué un parcours linéaire et direct vers une formation supérieure (HES essentiellement, mais parfois vers l'université via un raccordement), $5 \%$ ont quitté la formation (temporairement ou non) après avoir obtenu une MP, $9 \%$ sont en cours d'acquisition de leur MP (transition indirecte ou redoublement) et $15 \%$ étudient dans d'autres filières (essentiellement des écoles supérieures n'exigeant pas la MP). Au total, la moitié de cette population poursuit ou a terminé une formation post-CFC.

Après un CFC dual, la configuration est la même, mais dans des proportions différentes. Seuls $5 \%$ ont directement enchaîné une MP et une orientation vers une Haute école spécialisée ou l'université, $2 \%$ ont quitté la formation après avoir suivi une formation en MP, $4 \%$ y sont encore et $7 \%$ se sont dirigés vers une autre formation (surtout ES). Dix-huit mois après le CFC dual, 18 \% des diplômés poursuivent ou ont terminé une formation.

Le choix dual $v$ s plein-temps est en partie une question de contingences (métiers envisagés, places d'apprentissage disponibles, difficultés à se faire engager, conditions d'accès des écoles professionnelles), mais également de choix de rester dans un univers scolaire $v s$ d'intégrer une entreprise. Les jeunes en formation duale sont davantage intéressés par une transition rapide vers la vie active, probablement dès le début de leur formation. A l'inverse, un diplôme obtenu en école offre un contexte de formation plus proche des exigences d'une formation supérieure, via l'obtention d'une MP. C'est donc parmi les apprentis en école que l'on compte le plus de jeunes qui poursuivent leur formation après un CFC.

En étudiant l'échelon suivant (la MP), on constate le double usage de ce diplôme : ouvrir vers les études supérieures pour $54 \%$ des titulaires d'une MP (HES surtout, HEU et rarement ES) et s'insérer dans la vie active (39\%). Les titulaires d'une MP ont été interrogés sur les raisons qui les ont poussés à entreprendre cette formation. Les trois raisons principales sont : "pour continuer des études dans une HES", "parce que mes capacités me le permettent " et "pour améliorer mes chances de trouver un travail» (scores respectifs de 7.25, 7.06 et 6.75 sur une échelle de 9 points). Clairement, la MP est choisie tant pour préparer une transition vers la vie active que pour s'orienter vers des études supérieures. Comme pour le CFC, les jeunes qui ont obtenu leur MP intra-CFC en école sont davantage en formation que ceux qui l'ont obtenue en mode dual (respectivement $66 \%$ et $44 \%$, V de Cramer 0.23, $\mathrm{p}<0.01)$.

\section{Conclusion}

Un parcours de formation professionnelle au secondaire II ne se réduit pas à apprendre un métier que l'on va exercer sitôt le diplôme obtenu. Toute certification professionnelle initiale ou supplémentaire peut être convertie en outil d'insertion dans une formation ou sur le marché du travail.

Avec des systèmes de formation en partie différents, on retrouve les mêmes tendances dans les cantons de Vaud et de Genève, à deux nuances près : Genève connaît un marché du 
travail plus tendu et la formation professionnelle prend une place plus importante dans le canton de Vaud.

La situation des jeunes dix-huit mois après l'obtention du diplôme est modulée par une imbrication de facteurs, dont le niveau scolaire (l'exigence du diplôme), le mode de formation (en école ou en alternance) et les caractéristiques sociodémographiques des jeunes (genre, âge, provenance social, statut migratoire).

Une certification de moindre qualification engendre une transition à l'emploi plus difficile ; à l'opposé, une formation complémentaire au CFC (la MP) positionne plus favorablement les jeunes, tant pour une transition vers la vie active que dans une perspective de poursuite d'études supérieures. Un apprentissage dual, qui inclut l'expérience de l'entreprise, facilite l'accès à l'emploi, tandis que l'apprentissage effectué en école est davantage orienté vers la poursuite d'études. Ceci dans un contexte marqué par les inégalités sociales perceptibles lors du processus de transition post-diplôme.

Malgré les limites de l'enquête, qui ne couvre qu'une période de la transition vers la vie active, et dont l'aspect dynamique n'est que sommairement abordé, on perçoit des modes de transition, après le diplôme, souvent révisés, induisant des changements voulus ou subis, et un certain niveau d'incertitude. Cette diversité des situations (Pollien et Bonoli, 2012) brouille au premier abord le paysage de la formation et de l'insertion. Elle produit des transitions souvent complexes avec des tactiques d'accumulation, des stratégies de retour ou de "bifurcations tâtonnantes" (Berthet et al., 2008, p. 45). Mais, dans le même temps, une multiplication des solutions possibles crée des espaces de liberté et des marges de manœuvre, parfois limitées, qui permettent à certains jeunes de construire leurs parcours de formation sans renoncement préalable et à d'autres, qui se trouvent dans une situation difficile, de se tourner vers des solutions de requalification.

Durant la transition, les situations de formation, d'emploi, de recherche d'emploi ou d'autres activités se succèdent et souvent s'imbriquent. Travail durant la formation et formation continue en emploi sont fréquents (CSRE, 2014 ; OFS, 2009). En effet, dix-huit mois après leur diplôme, environ un tiers des jeunes qui poursuivent leur formation professionnelle déclarent également travailler (29\%) et un cinquième des jeunes en emploi disent fréquenter une formation continue.

La transition post-secondaire II apparaît alors comme une sorte de «tuilage » de la formation, du travail et des autres activités qui coexistent pour un temps dans cette phase mouvante de transition et d'orientation où la linéarité n'est pas (ou plus) le modèle dominant, préfigurant ainsi les nombreuses mobilités (OFS, 2012) qui composent de plus en plus les carrières professionnelles. 


\section{Bibliographie}

Bachmann Hunziker K., Leuenberger Zanetta S., Mouad R. et Rastoldo F. (2014), Que font les jeunes 18 mois après l'obtention de leur diplôme de niveau secondaire II ? Etat des lieux dans les cantons de Vaud et de Genève, SRED, URSP, Genève, Lausanne.

Beduwé C. et Fourcade B. (2014), "L'évaluation des formations par l'insertion professionnelle va-t-elle de soi ?", in Beduwé C., Bedin V. et Croity-Belz S. (Eds), Evaluation formation emploi. Un chantier pluridisciplinaire, L'Harmattan, Paris.

Berthelot J.-M. (1993), École, orientation, société, PUF, Paris.

Berthet T., Boudesseul G., Borras I., Coinaud C., Grelet, Y., Legay A., Romani C. et Vivent C. (2008), Valeur du diplôme. Place et rôle dans les parcours scolaires et professionnels, Céreq, Marseille.

Billet S. \& Johnson G. (2012), Experiences of school Transitions: Policies, Practice and Participant, Springer, Dordrecht.

Bloomer M. \& Hodfinson P. (2000), "Learning Careers: Continuity and Change in Young People’s Dispositions to Learning", British Educational Research Journal, 26(5), pp. 583-597.

Boudesseul G., Coinaud C., Grelet Y. et Vivent C. (2008), « Réversibilité et chemins de traverse en formation initiale ", in Cart B., Giret J.-F., Grelet Y. et Werquin P. (eds). Derrière les diplômes et certifications, les parcours de formation et leurs effets sur les parcours d'emploi, Céreq, Marseille, pp. 211-221.

Bourdieu P. et Passeron J.-C. (1970), La reproduction : éléments pour une théorie du système d'enseignement, Editions de Minuit, Paris.

Boutinet J.-P. (2007), « L'espace contradictoire des conduites à projets : entre le projet d'orientation du jeune et le parcours atypique de l'adulte ", L'orientation scolaire et professionnelle, 2007/36, $\mathrm{n}^{\circ}$ 1, pp. 19-32.

Boutinet J.-P. (2014), «Actualité des transitions et parcours de vie adulte ", L'orientation scolaire et professionnelle, 2014/43, n 4, pp. 397-412.

Cattaneo A., Donati M. et Galeandro Bocchino C. (2009), "Quinze ans en 1992, trente ans aujourd'hui ", Revue suisse des sciences de l'éducation, 31(2), pp. 229-248.

CDIP (2011), Déclaration 2011 sur les objectifs politiques communs concernant l'espace suisse de la formation, CDIP, Berne.

CEREQ (2012), Quand l'école est finie... Premiers pas dans la vie active d'une génération, Céreq, Marseille.

CSRE (Eds) (2014), L'éducation en Suisse, Rapport 2014, CSRE, Aarau. 
Davaud C., Mouad R. et Rastoldo F. (2010), Situation des diplômés de l'enseignement public genevois, 18 mois après l'obtention de leur titre, Volée 2007, SRED, Genève.

DEFR (2013), Encouragement ciblé et soutien des jeunes ayant des aptitudes diverses au moment de la transition I et pendant la formation professionnelle, Rapport du Conseil fédéral, Département fédéral de l'économie de la formation et de la recherche, Berne.

Deltand M. et Kaddouri M. (2014), « Les individus face à l'épreuve des transitions biographiques ", L'orientation scolaire et professionnelle, 2014/43, n 4, pp. 413-434.

De Broucker P., Gensbittel M. et Mainguet C. (2000), Déterminants scolaires et analyse de la transition, Service des études et de la statistique, Namur.

Dubar, C. (2001), "La construction sociale de l'insertion professionnelle ", Education et sociétés, 2001/1, nº 7 , pp. 3-36.

Dubet F. (1994), Sociologie de l'expérience, Seuil, Paris.

Doray P., Picard F., Trottier C. et Groleau A. (2009a), Les parcours éducatifs et scolaires. Quelques balises conceptuelles, vol. 44, Fondation canadienne des bourses d'études du millénaire, Montréal.

Doray P., Comoe E., Trottier C., Picard F., Murdoch J., Laplante B., Moulin S., MarcouxMoisan M., Groleau A. et Bourdon S. (2009b), Parcours scolaires et modes de transition dans l'enseignement postsecondaire canadien. Note 4 : Projet transitions (Vol. 45), Fondation canadienne des bourses d'études du millénaire, Montréal.

Fibbi R., Kaya B. et Piguet E. (2003), Le passeport ou le diplôme? Etude des discriminations à l'embauche des jeunes issus de la migration, Forum suisse pour l'étude des migrations et de la population, Neuchâtel.

Guichard J. (2013), «Comment aider les jeunes formés dans une école malade de l'orientation à s'orienter dans la vie ? ", L'orientation scolaire et professionnelle, 42 / 2013, pp. 181-198.

Hrizi Y, Mouad R., Petrucci F. et Rastoldo F. (2014), " Les parcours de formation des jeunes en difficultés scolaires à la fin du cycle d'orientation ", Note d'information du SRED, 65.

IHK Hessen (2015), Bundnis für Ausbildung in Hessen, Industrie und Handelkammer Arbeitsgemeinschaft Hessen, Wiesbaden.

Kammermann M., Balzer L. et Hättich A. (2013), « Des professionnels satisfaits de leur AFP », Panorama, 27(6), p. 16.

Keller A., Hupka-Brunner S. et Meyer T. (2010), Parcours de formation post-obligatoire en Suisse : les sept premières années, Unibasel, TREE, Bâle. 
Lamamra N., Fassa Recrosio F. et Chaponnière M. (2014), "Formation professionnelle : l'apprentissage des normes de genre ", Nouvelles Questions féministes, 33 (1), pp. 8-14.

Marcia J.-E. (1966), “Development and validation of ego-identity status,” Journal of personality and social psychology, 3, pp. 551-558.

Masdonati J. et Zittoun T. (2012), "Les transitions professionnelles : processus psychosociaux et implications pour le conseil en orientation ", L'orientation scolaire et professionnelle, 41 / 2012-2, pp. 229-253.

Meyer T., Hupka-Brunner S et Keller A. (2012), „Ausbildungs und Erwerbsverläufe des PISA 2000/TREE-Kohorte : Synopsis 2000-2007“, In Bergman M.-M., HupkaBrunner S., Keller A, Meyer T et Stalder B, (Eds), Transitions juvéniles en Suisse. Résultats de l'étude longitudinale TREE, Seismo, Zürich, pp. 86-91.

OCDE (1996), Regards sur l'éducation - Analyse, OCDE, Paris.

OFPC (2013), Développer et promouvoir la formation professionnelle et continue, Office pour l'orientation, la formation professionnelle et continue, Genève.

OFS (2014a), Système d'éducation - Examens. Récupéré le 23 juin 2014 de http://www. bfs.admin.ch/bfs/portal/fr/index/themen/15/02/data/blank/07.html\#Cubes

OFS (2014b), Cantons, communes - Population résidante permanente par canton. Récupéré le 23 juin 2014 de http://www.bfs.admin.ch/bfs/portal/fr/index/themen/01/02/blank/ key/raeumliche_verteilung/kantone_gemeinden.html

OFS (2014c), Résultats détaillés de la Statistique de l'emploi (STATEM). Récupéré le 23 juin 2014 de http://www.bfs.admin.ch/bfs/portal/fr/index/themen/03/02/blank/ data/02.html

OFS (2014d), Diplômes et compétences - Taux de diplômes du degré secondaire II. Récupéré le 23 juin 2014 de http://www.bfs.admin.ch/bfs/portal/fr/index/themen/15/17/blank/01. indicator.405101.4035.html

OFS (2014e), Chômeurs et taux de chômage au sens du BIT. Récupéré le 23 juin 2014 de http://www.bfs.admin.ch/bfs/portal/fr/index/themen/03/03/blank/key/erwer....

OFS (2009), Etudier sous Bologne. Rapport principal de l'enquête sur la situation sociale et économique des étudiant-e-s des hautes écoles suisses, OFS, Neuchâtel.

OFS (2012), La mobilité professionnelle. Une analyse sur la base de l'enquête suisse sur la population active (ESPA) de 1993 à 2011, OFS, Neuchâtel.

Padiglia S. (2005), Les transitions dans les itinéraires de formation, IRDP, Neuchâtel.

Padiglia S. (2007), "Itinéraires de transition et solutions transitoires en Suisse ", dans Behrens M. (dir.), La transition de l'école à la vie active ou le constat d'une problématique majeure, IRDP, Neuchâtel, pp. 13-21. 
Perez-Roux T. et Balleux A. (2014), "Transitions professionnelles désirées-contraintes : quelle dynamiques identitaires des acteurs à l'épreuve des contextes ? ", L'orientation scolaire et professionnelle, 2014/43, $\mathrm{n}^{\circ}$ 4, pp. 391-395.

Pollien A. et Bonoli L. (2012), Parcours de formation: analyse des trajectoires de formation des personnes résidant en Suisse, FORS Working Paper Series, paper 2012-2, FORS, Lausanne.

Rebsamen F. (2015), Le plan de relance de l'apprentissage, Gouvernement français, Paris.

Tap P. (1988), La société pygmalion?, Dunod, Paris. 